contended that the box in which Ross's tube was enclosed could not possibly account for the results he obtained. Prof. Gray described experiments on $\gamma$-rays and showed that they were consistent with the Compton theory. Prof. Raman made an eloquent appeal against a too hasty abandonment of the classical theory of scattering. Compton sketched an extension of his theory in which he considered not only free electrons but also those which were more tightly bound. He showed that the extended theory gave rise in the limiting case to the formula used by Duane and therefore embraced Duane's results. The fundamental difference between the two theories remains; Duane uses only the well-established quantum energy equation, while Compton in addition introduces the idea of conservation of momentum in the interaction between the radiation and matter. There are difficulties in the way of both theories, but at the present stage of the experimental work it is needless to dwell upon them. Before the theoretical side of the question can be satisfactorily discussed, further experimental work must be done. At the time of the meeting each observer appeared to have almost overwhelming evidence in favour of his point of view, and had the audience only had to listen to one side--either side would have done equally well-it would probably have been convinced as to the accuracy and soundness of the views advanced. As it was, however, the average member left the meeting inclined to echo the sentiments of the lover in the "Beggar's Opera" who sings,

" How happy could I be with either, Were t'other dear charmer away!"

\title{
The Spectrohelioscope. ${ }^{1}$
}

By Prof. George E. Hale, For. Mem. R.S.

M ONOCHROMATIC images of the sun, photographically recorded with the spectroheliograph, reveal the phenomena of the solar atmosphere in projection against the disk. The light from a spectral line of calcium, hydrogen, or other substance is singled out by a narrow second slit, which moves across the plate while the first slit moves across the solar image. A monochromatic picture is thus built up of countless narrow slit images, recorded side by side in slow succession.

The vortices and other significant structures thus disclosed, with the exception of an occasional brilliant eruption or unusually dark hydrogen flocculus, are beyond the reach of visual observation with the spectroscope. The simple method of opening the slit, which affords an excellent view of prominences at the sun's limb, because the light of the sky is sufficiently weakened by dispersion, is seriously limited when applied to the intensely brilliant disk. Even with the highest dispersion the slit cannot be opened sufficiently to reveal the characteristic structure of the dark hydrogen flocculi. Moreover, with a widened slit the image is not strictly monochromatic.

This simple expedient of rapidly oscillating the narrow spectroscope slit, and synchronously with it a second slit transmitting the $\mathrm{H} a$ line to the eye, was tried by the earliest observers of the prominences. With suitable precautions this device gives excellent images of prominences through persistence of vision, but it did not survive the introduction by Zoellner and Huggins of the wide slit method, and apparently was not tried for observing the sun's disk.

Many years ago I made some preliminary trials of the oscillating slit method with the 3o-foot spectroscope and 6o-foot tower telescope on Mount Wilson. I have only recently found opportunity to develop a satisfactory instrument based on this principle with which I have now secured good visual observations of both bright and dark flocculi.

An image of the sun two inches in diameter, given by a colostat and a I2-inch objective formerly belonging to the Kenwood Observatory, ${ }^{2 \times}$ was observed with a grating spectroscope mounted horizontally. In this instrument the light passing through the slit falls on a 6-inch concave mirror of 13 -feet focal length, which returns a parallel beam to a point just below the slit, where a 6 -inch plane grating is mounted. This sends the diffracted beam to a second 6-inch concave mirror, supported below the collimating mirror, which forms an image of the spectrum in the

1 Communicated to the National Academy of Sciences, Washington, on July 7, and published in the Proceedings of the Academy for August, vol. ro, No. 8 , 1924 .

2 Kindly loaned me by Prof. Frost. The apparatus was set up temporarily in my garden at South Pasadena. same plane as the first slit, and immediately below it. A fixed second slit at this point permits any part of the spectrum to be isolated. The grating was ruled by Jacomini with about I5,000 lines to the inch on the ruling machine of the Mount Wilson Observatory, with a diamond ground after Anderson's formula so as to give great brightness at the red end of the first order spectrum. The definition is perfect, and the brightness near $\mathrm{Ha}$ remarkable, as the attempt to concentrate most of the light in one spectrum and to favour the red end proved successful.

Suppose the first and second slits to be carried at opposite ends of a brass bar, mounted on a bearing half-way between them and thus free to revolve about this centre. Place the bar vertical, and turn the grating until the $\mathrm{Ha}$ line in the bright first order is bisected by the second slit. With the optical arrangement employed, a small displacement of the first slit to the right causes an exactly equal displacement of the $\mathrm{Ha}$ line to the left. Thus if the bar is oscillated back and forth by means of a small electric motor, a monochromatic image of the sun will be seen through a low power positive eyepiece focussed on the second slit.

This arrangement serves very well for the observation of prominences at the limb, where they can be seen at their full height with slits of moderate width. It also shows exceptionally bright or dark flocculi on the disk, though the slits must be narrower in order to give sufficient purity and reduce the brightness of the continuous spectrum. For flocculi of ordinary intensity the best results have been obtained with the aid of multiple slits, five at each end of the bar, 0.003 inch wide and 0.08 inch apart. A fixed slit, slightly less than 0.08 inch wide, must be used behind the upper slits, to prevent the formation of overlapping spectra. Two complete oscillations of the bar per second, corresponding to twenty illuminations of the retina, give a sufficiently steady image. A rotating disk, carrying a large number of radial slits, is in some respects a more satisfactory device for the same purpose.

This instrument, which may appropriately be called a spectrohelioscope, should prove a valuable auxiliary of the spectroheliograph in several kinds of work. It will permit the rapidly changing forms of eruptions on the disk to be followed visually, and be of special service in deciphering the curious differences of structure sometimes found on photographs of flocculi taken simultaneously with the opposite edges of $\mathrm{Ha}$. As the oscillating bar can be moved toward red or violet by a micrometer screw while observations are being made, the possibility of passing instantly from one edge of the line to the other should assist in the interpretation of the spectroheliograph results. 